\title{
LIE POWERS OF FREE MODULES FOR CERTAIN GROUPS OF PRIME POWER ORDER
}

\author{
R. M. BRYANT and I. C. MICHOS \\ To Laci Kovács on his 65th birthday
}

(Received 22 October 2000)

Communicated by R. A. Bryce

\begin{abstract}
Let $G$ be a finite group of order $p^{k}$, where $p$ is a prime and $k \geqslant 1$, such that $G$ is either cyclic, quaternion or generalised quaternion. Let $V$ be a finite-dimensional free $K G$-module where $K$ is a field of characteristic $p$. The Lie powers $L^{n}(V)$ are naturally $K G$-modules and the main result identifies these modules up to isomorphism. There are only two isomorphism types of indecomposables occurring as direct summands of these modules, namely the regular $K G$-module and the indecomposable of dimension $p^{k}-p^{k-1}$ induced from the indecomposable $K H$-module of dimension $p-1$, where $H$ is the unique subgroup of $G$ of order $p$. Formulae are given for the multiplicities of these indecomposables in $L^{n}(V)$. This extends and utilises work of the first author and R. Stöhr concerned with the case where $G$ has order $p$.
\end{abstract}

2000 Mathematics subject classification: primary 17B01; secondary $20 \mathrm{C} 20$.

\section{Introduction}

Let $G$ be a group, $K$ a field and $V$ a $K G$-module. Let $L(V)$ denote the free Lie algebra on $V$, that is, the free Lie algebra which contains $V$ as a subspace and which has every basis of $V$ as a free generating set. For each positive integer $n$, let $L^{n}(V)$ be the homogeneous component of degree $n$ in $L(V)$. The action of $G$ on $V$ extends naturally to $L(V)$, so that $G$ acts on $L(V)$ by Lie algebra automorphisms. In this way $L(V)$ becomes a $K G$-module with each $L^{n}(V)$ as a submodule, called the $n$th Lie power of $V$.

(c) 2001 Australian Mathematical Society 0263-6115/2001 \$A2.00+0.00 
Suppose now that $G$ is finite and $V$ is finite-dimensional. If the characteristic of $K$ is zero then each $L^{n}(V)$ is semisimple and the character of this module is given in terms of the character of $V$ by Brandt's character formula [3]. Thus $L^{n}(V)$ may be determined up to isomorphism, at least in principle, by the character orthogonality relations. In the case where $K$ has prime characteristic $p$, and $p$ divides the order of $G$, it is much more difficult to obtain information about the module structure of $L^{n}(V)$. Recent progress is described in [4] and [5]. The reader is also referred to [4] or [5] for further details of the background and underlying concepts.

The main result of the first author and Stöhr in [5] is a description of $L^{n}(V)$ in the case where $K$ has prime characteristic $p, G$ is cyclic of order $p$ and $V$ is a finitedimensional free $K G$-module. The module $L^{n}(V)$ decomposes into a direct sum of modules isomorphic either to the regular $K G$-module or to the indecomposable of dimension $p-1$. Here we extend this result to the case where $G$ is an arbitrary cyclic $p$-group or (when $p=2$ ) a quaternion or generalised quaternion group.

THEOREM 1. Let $G$ be a finite group of order $p^{k}$, where $p$ is a prime and $k \geqslant 1$, such that $G$ is either cyclic, quaternion or generalised quaternion. Let $H$ be the unique subgroup of $G$ of order $p$. Let $K$ be a field of characteristic $p$ and let $V$ be $a$ finite-dimensional free $K G$-module. Then, for each positive integer $n, L^{n}(V)$ is a direct sum of $r(n)$ copies of the regular KG-module and $s(n)$ copies of the indecomposable $K G$-module of dimension $p^{k}-p^{k-1}$ induced from the indecomposable $K H$-module of dimension $p-1$, where

$$
s(n)=-\frac{1}{n p^{k-1}} \sum_{\substack{d \\ p|d| n}} \mu(d)(\operatorname{dim} V)^{n / d}
$$

and $r(n)=p^{-k} \operatorname{dim} L^{n}(V)-\left(1-p^{-1}\right) s(n)$.

In the equation for $s(n), \mu$ is the Möbius function, $\operatorname{dim} V$ denotes the dimension of $V$ as a $K$-space, and the summation is over all positive divisors $d$ of $n$ which are divisible by $p$. The second equation yields $r(n)$ because of Witt's formula for the dimension of $L^{n}(V)$ :

$$
\operatorname{dim} L^{n}(V)=\frac{1}{n} \sum_{d \mid n} \mu(d)(\operatorname{dim} V)^{n / d} .
$$

Theorem 1 will be derived from the following more general result.

THEOREM 2. Let $G$ be a non-trivial finite $p$-group, where $p$ is a prime, and let $H$ be the subgroup generated by all elements of $G$ of order $p$. Let $K$ be a field of characteristic $p$ and let $V$ be a finite-dimensional free $K G$-module. Then, for each positive integer $n, L^{n}(V)$ is isomorphic to a module induced from some $K H$-module. 
Theorem 2 shows that there is a $K H$-module $U$ such that $L^{n}(V) \cong U \uparrow^{c}$. Thus, to find $L^{n}(V)$ up to isomorphism it is sufficient to find $U$. However,

$$
L^{n}\left(V \downarrow_{H}\right) \cong L^{n}(V) \downarrow_{H} \cong U \uparrow^{G} \downarrow_{H} .
$$

If we assume that $H$ is central in $G$ then $U \uparrow^{G} \downarrow_{H}$ is isomorphic to the direct sum of [ $G: H$ ] copies of $U$. Thus we can find $U$ up to isomorphism if we can find $L^{n}\left(V \downarrow_{H}\right)$. However, $V \downarrow_{H}$ is a free $K H$-module. Thus, when $H$ is central in $G$, Theorem 2 reduces the problem of finding Lie powers of free $K G$-modules to the same problem for $H$.

Under the assumptions of Theorem $1, H$ is central in $G$ and has order $p$. Thus Theorem 1 can be obtained from Theorem 2 by means of the main result of [5]. When $G$ is any finite abelian $p$-group, Theorem 2 makes a reduction to the case where $G$ is elementary abelian. At present, however, we are not able to deal with an elementary abelian $p$-group of order greater than $p$. The representation theory of such a group is complicated by the fact that it has infinitely many isomorphism types of indecomposable modules over a field of characteristic $p$.

The authors are grateful to Dr L. G. Kovács, Dr A. I. Papistas and Dr R. Stöhr for helpful discussions on the topics considered here. In particular, Dr Stöhr kindly pointed out a substantial simplification that could be made to our original proof of Lemma 3. The special case of Theorem 1 with $p=2$ was obtained in the second author's Ph.D. thesis [7] and the special case where $p=2$ and $G$ is cyclic was also obtained independently by A. I. Papistas in unpublished work.

\section{Preliminaries}

Throughout the paper $K$ denotes a field, and in this section $K$ is an arbitrary field. All Lie algebras are Lie algebras over $K$, and all tensor products are taken with respect to $K$. By a $K G$-module, where $G$ is a group, we mean a right module for the group algebra $K G$. As mentioned in Section 1, if $V$ is a $K G$-module then the free Lie algebra $L(V)$ acquires the structure of a $K G$-module.

For any Lie algebra $L,[u, v]$ denotes the product of elements $u$ and $v$ of $L$, and expressions of the form $\left[u_{1}, u_{2}, \ldots, u_{n}\right]$ denote left-normed products: thus, for $n \geqslant 3$,

$$
\left[u_{1}, u_{2}, \ldots, u_{n}\right]=\left[\left[u_{1}, \ldots, u_{n-1}\right], u_{n}\right] .
$$

For subspaces $U_{1}, U_{2}, \ldots, U_{n}$ of $L,\left[U_{1}, U_{2}, \ldots, U_{n}\right]$ denotes the subspace of $L$ spanned by all elements $\left[u_{1}, u_{2}, \ldots, u_{n}\right]$ with $u_{i} \in U_{i}$ for $i=1, \ldots, n$.

By a graded vector space over $K$ we mean a $K$-space $V$ with a distinguished $K$-space decomposition $V=V_{1} \oplus V_{2} \oplus \cdots$. A graded subspace of $V$ is then a subspace $W$ such that $W=\left(W \cap V_{1}\right) \oplus\left(W \cap V_{2}\right) \oplus \cdots$. 
Let $L$ be a free Lie algebra over $K$ and, for each positive integer $n$, let $L_{n}$ be the $n$th homogeneous component of $L$ with respect to a given free generating set for $L$. Then $L$ is a graded vector space, $L=L_{1} \oplus L_{2} \oplus \cdots$. A subalgebra of $L$ is called graded if it is graded as a subspace. By the theorem of Shirshov and Witt (see [8, Theorem 2.5]), every subalgebra of $L$ is free on some free generating set. If $Q$ is a subalgebra of $L$ and $W$ is a subspace of $L$ such that $Q$ is freely generated by a basis of $W$ we say (with slight abuse of language) that $Q$ is freely generated by $W$ and write $Q=L(W)$. In this case every basis of $W$ is a free generating set for $Q$. The notation $L(W)$ is used for the subalgebra of $L$ generated by a subspace $W$ only in the case where $L(W)$ is freely generated by $W$.

LEMMA 1. Let $Q$ be a graded subalgebra of the free Lie algebra $L$ and write $Q=Q_{1} \oplus Q_{2} \oplus \cdots$ where $Q_{i}=Q \cap L_{i}$ for all $i$. For $i \geqslant 1$ let $R_{i}$ be the subalgebra of $Q$ generated by $Q_{1} \oplus \cdots \oplus Q_{i}$ and let $R_{0}=0$. Let $W$ be a subspace of $Q$ which has the form $W=W_{1} \oplus W_{2} \oplus \cdots$, where $W_{i}=W \cap Q_{i}$ for all $i \geqslant 1$. Then $Q$ is freely generated by $W$ if and only if

$$
Q_{i}=\left(R_{i-1} \cap Q_{i}\right) \oplus W_{i} \quad \text { for all } i \geqslant 1 .
$$

ProOF. For the purposes of this proof, if $X$ is any subset of $L$ we write $K X$ for the subspace of $L$ spanned by $X$ and $\langle X\rangle_{L}$ for the Lie subalgebra of $L$ generated by $X$. For $i \geqslant 1$, let $E_{i}=Q_{1} \oplus \cdots \oplus Q_{i}$ and let $E_{0}=0$. Thus, for all $i \geqslant 1, R_{i-1}=\left\langle E_{i-1}\right\rangle_{L}$. Also, write $E_{i}^{\prime}=E_{i} \cap R_{i-1}$. Then

$$
E_{i}^{\prime}=Q_{1} \oplus \cdots \oplus Q_{i-1} \oplus\left(R_{i-1} \cap Q_{i}\right)
$$

If (2.1) holds, then any basis $X_{i}$ of $W_{i}$ is a basis for $E_{i}$ modulo $E_{i}^{\prime}$. Hence, by the proof of Theorem 2.5 of [8], $Q$ is freely generated by $X_{1} \cup X_{2} \cup \cdots$. Thus $Q$ is freely generated by $W$.

Conversely, suppose that $Q$ is freely generated by $W$. For each $i \geqslant 1$, let $X_{i}$ be a basis of $W_{i}$ and let $X=X_{1} \cup X_{2} \cup \cdots$. Since $Q=\langle X\rangle_{L}, Q$ is spanned by the set of all Lie monomials formed from elements of $X$. For $i \geqslant 1, Q_{i}$ is spanned by all such monomials which belong to $L_{i}$. Therefore,

$$
Q_{i}=\left\langle X_{1} \cup \cdots \cup X_{i-1}\right\rangle_{L} \cap L_{i}+K X_{i} .
$$

However, $X$ is a free generating set for $Q$, and so

$$
Q_{i}=\left\langle X_{1} \cup \cdots \cup X_{i-1}\right\rangle_{L} \cap L_{i} \oplus K X_{i} .
$$

Hence $Q_{j} \subseteq\left\langle X_{1} \cup \ldots \cup X_{j}\right\rangle_{L}$ for $j=1, \ldots, i-1$, and so $R_{i-1}=\left\langle X_{1} \cup \cdots \cup X_{i-1}\right\rangle_{L}$. Thus (2.1) follows from (2.2). 
For subspaces $U$ and $V$ of any Lie algebra, let $V \geq U$ denote the subspace defined by

$$
V \imath U=V+[V, U]+[V, U, U]+\cdots \text {. }
$$

The following lemma is a version of 'Lazard elimination' (see [2, Chapter 2, Section 2.9, Proposition 10]) and it appears in [4, Lemma 2.2].

LEMMA 2. Let $G$ be any group and let $U$ and $V$ be KG-modules. Consider the free Lie algebra $L(U \oplus V)$ as a $K G$-module. Then $U$ and $V>U$ freely generate free subalgebras $L(U)$ and $L(V, U)$, and there is a KG-module decomposition

$$
L(U \oplus V)=L(U) \oplus L(V, U) .
$$

Furthermore,

$$
V \imath U=V \oplus[V, U] \oplus[V, U, U] \oplus \cdots,
$$

and, for each $n \geqslant 0$, there is a KG-module isomorphism

$$
[V, \underbrace{U, \ldots, U}_{n}] \cong V \otimes \underbrace{U \otimes \cdots \otimes U}_{n}
$$

under which $\left[v, u_{1}, u_{2}, \ldots, u_{n}\right]$ corresponds to $v \otimes u_{1} \otimes u_{2} \otimes \cdots \otimes u_{n}$ for all $v \in V$ and all $u_{1}, \ldots, u_{n} \in U$.

We apply Lemma 2 to obtain two further results.

LEMMA 3. Let $G$ be any group and let $V_{1}, \ldots, V_{m}$ be KG-modules, where $m$ is a positive integer. Consider the free Lie algebra $L\left(V_{1} \oplus \cdots \oplus V_{m}\right)$ as a $K G$-module. Let $Q$ be the ideal of $L\left(V_{1} \oplus \cdots \oplus V_{m}\right)$ generated by the subspaces $\left[V_{i}, V_{j}\right]$ with $i \neq j$. Then there is a KG-module decomposition

$$
L\left(V_{1} \oplus \cdots \oplus V_{m}\right)=L\left(V_{1}\right) \oplus \cdots \oplus L\left(V_{m}\right) \oplus Q .
$$

Furthermore, $Q$ is a free Lie subalgebra of $L\left(V_{1} \oplus \cdots \oplus V_{m}\right)$ of the form $Q=L(W)$, where $W=W_{2} \oplus W_{3} \oplus \cdots$ such that, for each $n \geqslant 2, W_{n}$ is a KG-submodule of $L^{n}\left(V_{1} \oplus \cdots \oplus V_{m}\right)$ equal to the direct sum of all subspaces $\left[V_{i_{1}}, V_{i_{2}}, \ldots, V_{i_{n}}\right]$ with $i_{1}>i_{2} \leqslant i_{3} \leqslant \cdots \leqslant i_{n}$. Furthermore, $\left[V_{i_{1}}, V_{i_{2}}, \ldots, V_{i_{n}}\right]$ is isomorphic to $V_{i_{1}} \otimes V_{i_{2}} \otimes \cdots \otimes V_{i_{n}}$ as a KG-module.

ProOF. By Lemma 2,

$$
L\left(V_{1} \oplus \cdots \oplus V_{m}\right)=L\left(V_{1}\right) \oplus L\left(Z_{1}\right),
$$


where $Z_{1}$ is the direct sum of the subspaces $V_{2}, \ldots, V_{m}$ and the subspaces

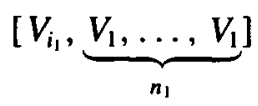

with $i_{1}>1$ and $n_{1}>0$. Also,

$$
[V_{i_{1}}, \underbrace{V_{1}, \ldots, V_{1}}_{n_{1}}] \cong V_{i_{1}} \otimes \underbrace{V_{1} \otimes \cdots \otimes V_{1}}_{n_{1}}
$$

as $K G$-modules. Using the summand $V_{2}$ of $Z_{1}$ we obtain $L\left(Z_{1}\right)=L\left(V_{2}\right) \oplus L\left(Z_{2}\right)$, where $Z_{2}$ is the direct sum of the subspaces $V_{3}, \ldots, V_{m}$ and the subspaces

$$
[V_{i_{1}}, \underbrace{V_{1}, \ldots, V_{1}}_{n_{1}}, \underbrace{V_{2}, \ldots, V_{2}}_{n_{2}}]
$$

with either $i_{1}>1, n_{1}>0, n_{2} \geqslant 0$ or $i_{1}>2, n_{1}=0, n_{2}>0$. Also

$$
[V_{i_{1}}, \underbrace{V_{1}, \ldots, V_{1}}_{n_{1}}, \underbrace{V_{2}, \ldots, V_{2}}_{n_{2}}] \cong V_{i_{1}} \otimes \underbrace{V_{1} \otimes \cdots \otimes V_{1}}_{n_{1}} \otimes \underbrace{V_{2} \otimes \cdots \otimes V_{2}}_{n_{2}}
$$

as $K G$-modules. Continuing in this way we obtain

$$
L\left(V_{1} \oplus \cdots \oplus V_{m}\right)=L\left(V_{1}\right) \oplus \cdots \oplus L\left(V_{m}\right) \oplus L(W),
$$

where $W$ is the direct sum of the subspaces $\left[V_{i_{1}}, V_{i_{2}}, \ldots, V_{i_{n}}\right]$ with $n \geqslant 2$ and $i_{1}>$ $i_{2} \leqslant i_{3} \leqslant \cdots \leqslant i_{n}$. Also,

$$
\left[V_{i_{1}}, V_{i_{2}}, \ldots, V_{i_{n}}\right] \cong V_{i_{1}} \otimes V_{i_{2}} \otimes \cdots \otimes V_{i_{n}}
$$

as $K G$-modules. Clearly, $\left[V_{i_{1}}, V_{i_{2}}, \ldots, V_{i_{n}}\right]$ is a submodule of $L^{n}\left(V_{1} \oplus \cdots \oplus V_{m}\right)$. Thus $W$ can be written in the required form.

It remains to show that $L(W)=Q$, where $Q$ is defined as in the statement of the lemma. Clearly $L(W) \subseteq Q$. Let $D$ be the Lie algebra direct sum,

$$
D=L\left(V_{1}\right) \oplus \cdots \oplus L\left(V_{m}\right),
$$

formed from the Lie algebras $L\left(V_{1}\right), \ldots, L\left(V_{m}\right)$ with componentwise multiplication. Let

$$
\pi: L\left(V_{1} \oplus \cdots \oplus V_{m}\right) \longrightarrow D
$$

be the Lie algebra homomorphism given by $u \pi=u$ for all $u \in V_{1} \cup \cdots \cup V_{m}$ : this exists because $L\left(V_{1} \oplus \cdots \oplus V_{m}\right)$ is free on $V_{1} \oplus \cdots \oplus V_{m}$. As is well known, $Q$ is the kernel of $\pi$. The restriction of $\pi$ to the subspace $L\left(V_{1}\right) \oplus \cdots \oplus L\left(V_{m}\right)$ of $L\left(V_{1} \oplus \cdots \oplus V_{m}\right)$ is clearly one-one and onto. Hence

$$
L\left(V_{1} \oplus \cdots \oplus V_{m}\right)=L\left(V_{1}\right) \oplus \cdots \oplus L\left(V_{m}\right) \oplus Q .
$$

Since $L(W) \subseteq Q$ we obtain $L(W)=Q$ by $(2.3)$. 
LEMMA 4. Let $G$ be any group and let $V$ be a KG-module. Let $Q$ be a subalgebra of $L(V)$ which has the form $Q=L\left(U_{r} \oplus U_{r+1} \oplus \cdots\right)$ for some positive integer $r$, where, for each $i \geqslant r, U_{i}$ is a free $K G$-submodule of $L^{i}(V)$. Let $n$ be a positive integer such that $n \geqslant r$. Then, for each $i \geqslant r$, there exists a free $K G$-submodule $X_{i}$ of $L^{i}(V)$ such that $Q=L\left(X_{r}\right) \oplus L\left(X_{r+1}\right) \oplus \cdots \oplus L\left(X_{n}\right) \oplus L\left(X_{n+1} \oplus X_{n+2} \oplus \cdots\right)$.

PROOF. By Lemma 2,

$$
L\left(U_{r} \oplus U_{r+1} \oplus \cdots\right)=L\left(U_{r}\right) \oplus L\left(\left(U_{r+1} \oplus U_{r+2} \oplus \cdots\right) 2 U_{r}\right) .
$$

Also, $\left(U_{r+1} \oplus U_{r+2} \oplus \cdots\right)$; $U_{r}$ is the direct sum of the modules $U_{r+i}<U_{r}$ for $i \geqslant 1$, and $U_{r+i} 2 U_{r}$ is the direct sum of the modules

$$
[U_{r+i}, \underbrace{U_{r}, \ldots, U_{r}}_{m}]
$$

for $m \geqslant 0$. Furthermore,

$$
[U_{r+i}, \underbrace{U_{r}, \ldots, U_{r}}_{m}] \cong U_{r+i} \otimes \underbrace{U_{r} \otimes \cdots \otimes U_{r}}_{m},
$$

so this module is a free $K G$-submodule of $L^{r+i+m r}(V)$. Thus we may write

$$
L\left(U_{r} \oplus U_{r+1} \oplus \cdots\right)=L\left(U_{r}\right) \oplus L\left(V_{r+1} \oplus V_{r+2} \oplus \cdots\right),
$$

where, for $i \geqslant 1, V_{r+i}$ is a free $K G$-submodule of $L^{r+i}(V)$. The result follows by induction on $n-r$.

\section{Main results}

Let $G$ be a non-trivial finite $p$-group, where $p$ is a prime, and let $H$ be the subgroup generated by all elements of $G$ of order $p$. Let $K$ be a field of characteristic $p$. For any $K G$-module $V$ we write $V \downarrow_{H}$ for the $K H$-module obtained by restriction, and for any $K H$-module $U$ we write $U \uparrow^{G}$ for the $K G$-module obtained by induction. We say that a $K G$-module is $H$-induced if it is isomorphic to a module induced from a $K H$-module. Clearly any direct sum of $H$-induced modules is $H$-induced.

ProOF OF THEOREM 2. Let $V$ be a finite-dimensional free $K G$-module. Write $L=L(V)$ and $L_{n}=L^{n}(V)$ for all $n \geqslant 1$. We must prove that $L_{n}$ is $H$-induced for all $n$. We use induction on $n$. Since $L_{1}=V$, the module $L_{1}$ is free. Thus it is induced from a free $K H$-module. Hence we may assume that $n \geqslant 2$ and that the result is true for all smaller values of $n$. 
Let $m$ be the index of $H$ in $G$ and let $\left\{c_{1}, \ldots, c_{m}\right\}$ be a (right) transversal for $H$ in $G$, where $c_{1}=1$. Since $V$ is a free $K G$-module, there is a subspace $Y$ of $V$ such that $V=Y(K G) \cong Y \otimes K G$. Write $V_{i}=Y(K H) c_{i}$ for $i=1, \ldots, m$. Then, since $H$ is normal in $G$, each $V_{i}$ is a free $K H$-module isomorphic to $V_{1}$. Also, $V=V_{1} \oplus \cdots \oplus V_{m}$ and $V \cong V_{1} \uparrow^{G}$.

By Lemma 3 applied to the group $H$, we have a $K H$-module decomposition $L=M \oplus Q$, where $M=L\left(V_{1}\right) \oplus \cdots \oplus L\left(V_{m}\right)$ and $Q$ is the ideal of $L$ generated by the subspaces $\left[V_{i}, V_{j}\right]$ with $i \neq j$. The subspaces $\left[V_{i}, V_{j}\right]$ are permuted under the action of $G$. Therefore $Q$ is a $K G$-submodule of $L$. Also, $L\left(V_{i}\right)=L\left(V_{1}\right) c_{i}$ for $i=1, \ldots, m$, so that $M$ is a $K G$-submodule of $L$. By Lemma 3, $Q=L(W)$ where $W$ is a graded subspace of $L$. Thus $Q$ is a graded subalgebra of $L$. Clearly $M$ is a graded subspace of $L$. It follows that $L_{n}=\left(L_{n} \cap M\right) \oplus\left(L_{n} \cap Q\right)$. Thus it suffices to show that $L_{n} \cap M$ and $L_{n} \cap Q$ are $H$-induced.

It is easily verified that $L^{n}\left(V_{i}\right)=L^{n}\left(V_{1}\right) c_{i}$ for $i=1, \ldots, m$ and

$$
L_{n} \cap M=L^{n}\left(V_{1}\right) \oplus \cdots \oplus L^{n}\left(V_{m}\right)
$$

It follows that $L_{n} \cap M \cong L^{n}\left(V_{1}\right) \uparrow^{G}$. Therefore $L_{n} \cap M$ is $H$-induced.

By Lemma 3, $W=W_{2} \oplus W_{3} \oplus \cdots$ where, for $i \geqslant 2, W_{i}$ is a free $K H$-submodule of $L_{i}$. We also write $W_{1}=0$. Since $Q$ is a graded subalgebra of $L$, it has the form $Q=Q_{1} \oplus Q_{2} \oplus \cdots$ where $Q_{i}=Q \cap L_{i}$ for all $i$. Clearly $Q_{1}=0$. For $i \geqslant 1$ let $R_{i}$ be the subalgebra of $Q$ generated by $Q_{1} \oplus \cdots \oplus Q_{i}$ and let $R_{0}=0$. Since $Q$ is a $K G$-module, $Q_{i}$ is a $K G$-module for each $i \geqslant 1$, and $R_{i-1} \cap Q_{i}$ is a submodule. Thus $Q_{i} /\left(R_{i-1} \cap Q_{i}\right)$ is a $K G$-module.

By Lemma $1, Q_{i}=\left(R_{i-1} \cap Q_{i}\right) \oplus W_{i}$ for all $i \geqslant 1$. Since $W_{i}$ is a free $K H$ module, $\left(Q_{i} /\left(R_{i-1} \cap Q_{i}\right)\right) \downarrow_{H}$ is a free $K H$-module. Therefore, by the definition of $H,\left(Q_{i} /\left(R_{i-1} \cap Q_{i}\right)\right) \downarrow_{E}$ is a free $K E$-module for every elementary abelian subgroup $E$ of $G$. Therefore, by a theorem of Chouinard, [1, Theorem 5.2.4], $Q_{i} /\left(R_{i-1} \cap Q_{i}\right)$ is a projective $K G$-module. Since $G$ is a $p$-group, this module is free (see, for example, [6, Theorem VII.7.15]). Therefore, for each $i \geqslant 1$, there is a $K G$-submodule $U_{i}$ of $Q_{i}$ such that $U_{i}$ is free and $Q_{i}=\left(R_{i-1} \cap Q_{i}\right) \oplus U_{i}$. Note that $U_{1}=0$ and write $U=U_{2} \oplus U_{3} \oplus \cdots$. By Lemma $1, Q$ is freely generated by $U$, that is $Q=L(U)$.

By Lemma 4, we may write

$$
Q=L\left(X_{2}\right) \oplus L\left(X_{3}\right) \oplus \cdots \oplus L\left(X_{n}\right) \oplus L\left(X_{n+1} \oplus X_{n+2} \oplus \cdots\right)
$$

where each $X_{i}$ is a free $K G$-submodule of $L_{i}$. It follows that $L_{n} \cap Q$ is the direct sum of the modules $L^{n / d}\left(X_{d}\right)$ where $d$ ranges over the divisors of $n$ in the set $\{2, \ldots, n\}$. By the inductive hypothesis each summand is $H$-induced. Therefore $L_{n} \cap Q$ is $H$-induced, as required. 
ProOf OF THEOREM 1. Now let $G$ have order $p^{k}$, with $k \geqslant 1$, and suppose that $G$ is either cyclic, quaternion or generalised quaternion. Thus the subgroup $H$ is central and is cyclic of order $p$. We write $J_{p}$ to denote a regular $K H$-module and $J_{p-1}$ to denote an indecomposable $K H$-module of dimension $p-1$ (this is isomorphic to the augmentation ideal of $K H$ ).

Let $V$ be a finite-dimensional free $K G$-module and let $n$ be a positive integer. By Theorem 2, there exists a $K H$-module $U$ such that $L^{n}(V) \cong U \uparrow^{G}$. Therefore

$$
L^{n}\left(V \downarrow_{H}\right) \cong L^{n}(V) \downarrow_{H} \cong U \uparrow^{G} \downarrow_{H}
$$

Since $H$ is central in $G, U \uparrow^{G} \downarrow_{H}$ is isomorphic to the direct sum of $p^{k-1}$ copies of $U$. However $V \downarrow_{H}$ is a free $K H$-module. Therefore, by [5, Theorem 1], $L^{n}\left(V \downarrow_{H}\right)$ is isomorphic to the direct sum of $r_{n}$ copies of $J_{p}$ and $s_{n}$ copies of $J_{p-1}$ where

$$
s_{n}=-\frac{1}{n} \sum_{\substack{d \\ p ! d \mid n}} \mu(d)(\operatorname{dim} V)^{n / d}
$$

and $p r_{n}+(p-1) s_{n}=\operatorname{dim} L^{n}(V)$. It follows that $U$ is isomorphic to the direct sum of $p^{-(k-1)} r_{n}$ copies of $J_{p}$ and $p^{-(k-1)} s_{n}$ copies of $J_{p-1}$. Therefore the induced module $L^{n}(V)$ is isomorphic to the direct sum of $p^{-(k-1)} r_{n}$ copies of $J_{p} \uparrow^{G}$ and $p^{-(k-1)} s_{n}$ copies of $J_{p-1} \uparrow^{G}$.

Clearly $J_{p} \uparrow^{G}$ is a regular $K G$-module and is indecomposable (see [6, VII.5.2]). Since $J_{p-1}$ remains indecomposable under any field extension it follows from Green's indecomposability theorem (see $[6, \mathrm{VII} .16 .6]$ ) that $J_{p-1} \uparrow^{G}$ is an indecomposable $K G$-module. Clearly it has dimension $p^{k}-p^{k-1}$. In the notation of Theorem 1 , $r(n)=p^{-(k-1)} r_{n}$ and $s(n)=p^{-(k-1)} s_{n}$. This gives Theorem 1 .

\section{References}

[1] D. J. Benson, Representations and cohomology II (Cambridge University Press, Cambridge, 1991).

[2] N. Bourbaki, Lie groups and Lie algebras, Part I: Chapters 1-3 (Hermann, Paris, 1975).

[3] A. Brandt, 'The free Lie ring and Lie representations of the full linear group', Trans. Amer. Math. Soc. 56 (1944), 528-536.

[4] R. M. Bryant, L. G. Kovács and R. Stöhr, 'Lie powers of modules for groups of prime order', Proc. London Math. Soc. (3), to appear.

[5] R. M. Bryant and R. Stöhr, 'On the module structure of free Lie algebras', Trans. Amer. Math. Soc. 352 (2000), 901-934.

[6] B. Huppert and N. Blackburn, Finite groups I/ (Springer, Berlin, 1982).

[7] I. C. Michos, Finite groups acting on free Lie algebras (Ph.D. Thesis, Manchester, 1998).

[8] C. Reutenauer, Free Lie algebras (Clarendon Press, Oxford, 1993). 
UMIST

LIAFA

PO Box 88

Université Paris 7

Manchester M60 1QD

75251 Paris

England

France

e-mail: bryant@umist.ac.uk

e-mail: michos@liafa.jussieu.fr 